\title{
Cognitive Change in Donepezil Treated Patients with Vascular or Mixed Dementia
}

\author{
Kenneth Rockwood, Arnold Mitnitski, Sandra E. Black, Matthew Richard, \\ Isabelle Defoy, on behalf of the VASPECT study investigators.
}

\begin{abstract}
Background: Vascular dementia (VaD) and mixed Alzheimer's disease (AD/VaD) are common. How best to monitor treatment is not clear. Our objective was to compare responsiveness and construct validity of change scores, following donepezil treatment, of the standardized Mini-Mental State Examination (sMMSE) and other measures potentially usable in primary care. Methods: A six-month, outcome measurement study. The Disability Assessment for Dementia (DAD), CLOX-1 and 2, Phonetic Fluency, a short Neuropsychiatric Inventory, (the NPI-Q), Clinical Global Impression (CGI) and the SymptomGuide ${ }^{\mathrm{TM}}$ (SG) were measured. Construct validity was tested by correlating change scores, and responsiveness by calculating standardized response means (SRMs). Results: Of 148 treated patients, 116 completed. The mean sMMSE increased by $0.7(95 \%$ Confidence Interval $(\mathrm{CI})=-0.005$, $1.41 ; \mathrm{p}=0.06 ; \mathrm{SRM}=0.15)$. There was no statistically significant difference in the DAD. The NPI-Q $(-1.4 ; 95 \% \mathrm{CI}=-2.08,-0.72$; $\mathrm{p}<0.01 ; \mathrm{SRM}=0.24), \mathrm{CLOX}-1(0.9 ; 95 \% \mathrm{CI}=0.19,1.61 ; \mathrm{p}<0.01 ; \mathrm{SRM}=0.21), \mathrm{CLOX}-2(0.9 ; 95 \% \mathrm{CI}=0.17,1.63 ; \mathrm{p}=0.03 ; \mathrm{SRM}=0.26)$, Phonetic Fluency $(0.9 ; 95 \% \mathrm{CI}=0.19,1.61 ; \mathrm{p}=0.02 ; \mathrm{SRM}=0.21)$ and $\mathrm{SG}(0.35 ; 95 \% \mathrm{CI}=0.20,0.51 ; \mathrm{p}<0.01 ; \mathrm{SRM}=0.28)$ each detected significant improvement. The CGI suggested improvement in 74 completers (64\%) - mostly "minimal" (44/116, 38\%) - while 21/116 $(18 \%)$ were worse. Change scores at 24 weeks were at best modestly correlated with each other (range -0.22 to 0.30 ). Discussion: Different measures showed different responsiveness, in a setting in which the mean treatment effect seems to have been small, but clinically detectable. Patient-centered and executive function measures might be useful in vascular and mixed dementia.
\end{abstract}

RÉSUMÉ: Changements cognitifs chez les patients atteints de démence vasculaire ou mixte traités par le donépézil. Contexte : La démence vasculaire (DV) et la maladie d'Alzheimer mixte (MA/DV) sont des maladies fréquentes. Toutefois, on ne connaît pas quelle est la meilleure façon de faire le suivi de ces patients au cours du traitement. Le but de notre étude était de comparer la réactivité et la validité conceptuelle des taux de changement observés au mini examen de l'état mental (MMSE) et à d'autres instruments de mesure utilisables dans les soins de première ligne, suite au traitement par le donépézil. Méthode : Nous avons effectué une étude des résultats obtenus après six mois de traitement. Nous avons utilisé l'échelle d'évaluation de l'invalidité associée à la démence (DAD), les tests du cadran de l'horloge CLOX-1 et 2, le test de la fluence verbale phonologique (TFVP), un inventaire neuropsychiatrique abrégé, le NPI-Q, le test d'impression clinique globale CGI et le Guide des symptômes (GS). La validité conceptuelle a été évaluée au moyen de la corrélation des scores de changement et la réponse au traitement a été évaluée par le calcul des moyennes des réponses standardisées (MRS). Résultats : Parmi les 148 patients traités, 116 ont complété les évaluations. La moyenne au MMSE a augmenté de 0,7 (IC à 95\% : - 0,005 à 1,41; p = 0,06; MRS = 0,15). Nous n'avons pas noté de différence significative au point de vue statistique pour le DAD. Le NPIQ (-1,4; IC à 95\% : -2,08 à -0,72; p < 0,01; MRS 0,24), le CLOX-1 (0,9; IC à 95\%: 0,19 à 1,61; p < 0,01; MRS 0,21), le CLOX-2 (0,9; IC à 95\% : 0,17 à 1,$63 ; \mathrm{p}=0,03 ;$ MRS = 0,26), le TFVP $(0,0$ IC à $95 \%: 0,19$ à 1,$61 ; \mathrm{p}=0,02 ;$ MRS = 0,21) et GS $(0,35 ;$ IC à $95 \%: 0,20$ à 0,$51 ;<0,01 ;$ MRS $=$ $0,28)$ ont tous détecté une amélioration significative. Le CGI suggérait une amélioration chez 74 sujets (64\%) et cette amélioration était minime (44/116, $38 \%$ ), alors que 21/116 étaient pires (18). Les scores de changement à 24 semaines étaient au mieux corrélés faiblement entre eux (écart de -0,22 à 0,30). Discussion : Différentes mesures ont montré une réactivité différente dans le contexte d'un effet moyen du traitement qui semble avoir été faible mais détectable au point de vue clinique. Des mesures centrées sur le patient et des mesures de la fonction exécutive pourraient être utiles dans la démence vasculaire et la démence mixte.

Can J Neurol Sci. 2013; 40: 564-571

Patients with Vascular Cognitive Impairment (VCI) constitute an important part of the burden of cognitive disorders in older adults. The VCI category represents a heterogeneous group of disorders that includes people who do not meet dementia criteria (Vascular Cognitive Impairment - No Dementia; VCI-ND) and those who do. ${ }^{1,2}$ Of those with dementia, both "pure" vascular dementia $(\mathrm{VaD})$ and mixed $\mathrm{VaD}$ /Alzheimer disease (AD) are recognized. The former is likely uncommon; the NINDS-AIREN VaD criteria $^{3}$ set a very high threshold for the detection of $\mathrm{VaD}$ and their "probable VaD" classification comes as close clinically to detecting "pure" VaD as the present state of diagnosis permits. ${ }^{1}$ Mixed dementia, however, is non-controversially recognized as common, including in clinical trials of Alzheimer disease,${ }^{4}$ even if the

From DGI Clinical Inc (KR, AM, MR); Division of Geriatric Medicine (KR, AM), Dalhousie University, Halifax, Nova Scotia; Division of Neurology (SEB), University of Toronto, Sunnybrook Health Sciences Centre, Toronto, Ontario; Pfizer Canada Inc. (ID), Kirkland, Quebec, Canada.

Received October 30, 2012. Final Revisions Submitted January 3, 2013. Correspondence to: Kenneth Rockwood, DGI Clinical Inc., Suite 208, 1344 Summer Street, Halifax, Nova Scotia, B3H 0A8, Canada. Email: krockwood@dgiclinical.com. 
extent to which vascular lesions give rise to dementia in the face of traditional AD markers is disputed..$^{5-9}$

The treatment of $\mathrm{VaD}$ and mixed $\mathrm{AD} / \mathrm{VaD}$ with cholinesterase inhibitors, although generally recommended, ${ }^{10-12}$ can be controversial. Sceptics point to the small size of the treatment effects in the two major clinical trials of donepezil in VaD. ${ }^{13,14}$ More recent work, which attempted to learn from the two trials, likewise was inconclusive. While patients with $\mathrm{VaD}$ who were treated with donepezil showed improvement on a neuropsychological test battery - Vascular-Alzheimer Disease Assessment Scale-Cognitive Subscale ${ }^{15}$ - this was not reflected in the clinical assessment. ${ }^{16}$ In consequence, the 4th Canadian Consensus Conference on the Diagnosis and Treatment of Dementia recommended treatment of patients with possible Alzheimer's disease and a cerebrovascular component, but not pure vascular dementia. ${ }^{17}$

Although it might simply be that donepezil does not much help people with vascular and mixed dementia, ${ }^{18}$ the small treatment effect might also reflect the insensitivity of commonly used outcome measures to detect change, especially if subtle effects on executive function are present. ${ }^{1,11,19}$ For this reason, there was interest in evaluating both commonly employed clinical trial outcome measures, as well as measures that might better detect executive function and thereby be more sensitive to clinically important changes in VCI. Our objectives were: to evaluate, in an open label clinical trial, whether the standardized Mini-Mental State Examination (sMMSE) captured any impact of donepezil on general cognition in primary care patients with clinico-radiographically defined $\mathrm{VaD}$ and mixed $\mathrm{AD} / \mathrm{VaD}$, and; to evaluate the responsiveness and construct validity of measures of executive dysfunction, behaviour, and activities of daily living (ADLs). Finally, as with any compound, we studied the safety and tolerability of donepezil in subjects with these types of dementia.

\section{MethodS}

\subsection{Patients, setting, sample size and selection criteria}

This was a six-month, open-label study, conducted between June 2005 and April 2008 at 30 primary care clinics across Canada. Treatment was with $5 \mathrm{mg} / \mathrm{d}$ donepezil for six weeks, to be increased to $10 \mathrm{mg} / \mathrm{d}$ for the following weeks; adjustable dosing was permitted. Patients were first screened and then examined at a separate baseline visit within one week, and then at 6,12 , and 24 weeks post baseline.

We expected a mean improvement on the sMMSE of 0.73 points at six months, with a standard deviation of 3.5.20,21 A twosided $\alpha=0.05$ t-test required 205 patients for $85 \%$ power to detect a significant improvement from baseline; with an anticipated drop-out rate of approximately $20 \%$, the plan was to enrol 260 patients in the study.

To be eligible for inclusion, patients had to meet both clinical and radiographic criteria. The clinical inclusion criteria, in addition to written informed consent by the patient and a primary caregiver, were: 50 years-of-age or older, with a reliable caregiver; $\mathrm{VaD}$ and/or mixed $\mathrm{AD} / \mathrm{VaD}$ diagnosed using DSM-IVTR criteria; ${ }^{22}$ mild-moderate severity, operationalized in people with a Hachinski Ischemia Score $>4^{23}$ and a Functional Assessment Staging Tool (FAST) ${ }^{24}$ score of 4 or 5. Radiographic inclusion criteria specified lesions compatible with a diagnosis of cerebrovascular pathology: e.g., the presence of one strategic (e.g., thalamic) or more than one infarct, two or more lacunes, or significant leukoaraiosis (e.g., extending $>3 \mathrm{~mm}$ from the ventricles). ${ }^{25}$ Patients were excluded if they had recently participated in other studies, were clinically unstable (including a systolic blood pressure $>160 \mathrm{mmHg}$ and/or diastolic blood pressure $>95 \mathrm{mmHg}$ ) or had a history of intolerance or hypersensitivity to donepezil.

\subsection{Measures}

Data were recorded on patient and caregiver demographics, the patient's medical history, general and neurological examination, vital signs, electrocardiogram (ECG), serum chemistry, hematology and urinalysis and concomitant medications. Vascular risk factors included hypertension, diabetes, hypercholesterolemia, transient ischemic attack (TIA), stroke, coronary artery disease, atrial fibrillation, smoking and body mass index. The six-item Hamilton Depression Scale (HAM_D-6) was used for screening. ${ }^{26}$

The primary efficacy measure was the 30-point sMMSE (a higher score is better). ${ }^{27}$ Other measures were chosen for their familiarity and/or potential feasibility for use in Canadian primary care settings. Activities of daily living were measured using the Disability Assessment for Dementia (DAD), which includes assessment of planning, initiation and execution of basic and instrumental activities (a higher score is better). ${ }^{28}$ Executive function was assessed using two variants of the clock drawing tests, CLOX-1 and CLOX-2 (a higher score is better) ${ }^{29}$ and the Phonetic letter fluency test (a higher score is better) ${ }^{30} \mathrm{In}$ the CLOX test, two clocks are drawn, one free hand (CLOX 1) and one copied (CLOX 2). Each CLOX test was scored separately. Phonetic letter fluency is a measure of how many words a subject can generate in one minute. The total number of words constituted the score. To assess behavior we used a brief form of the Neuropsychiatric Inventory, the NPI-Q. ${ }^{31}$ The brief NPI-Q is used to measure severity of behavioral manifestations of dementia together with the level of distress that each symptom causes the main caregiver. The 10-item NPI-Q is based on: delusions, hallucinations, agitation/aggression, depression/ dysphoria, anxiety, elation/euphoria, apathy/indifference, disinhibition, irritability/lability, and aberrant motor behavior. The 12-item version (used here) adds sleep and appetite/eating disorders. These scores are each rated as 0 (absent); 1 (mild); 2 (moderate) or; 3 (severe) giving a range from 0-36. The NPI-Q also measures the caregiver distress associated with each symptom, ranging from 0 (no distress) to 5 (very severe), so that the NPI-Q distress (NPI-Q-D) score ranges from 0 to 60 . Lastly, global functioning was measured using the Clinical Global Impression (CGI). The CGI measures a physician's global impression of a subject's clinical condition, at baseline in terms of severity (CGI-S), and at follow-up as interval change from baseline (CGI-I). At baseline, the subject was rated on a scale ranging from 1 (normal, not at all ill) to 7 (among the most extremely ill patients). At follow-up visits, the subject was rated on a 7-point scale ranging from 1 (very much improved) to 7 (very much worse).

In this study, the SymptomGuide ${ }^{\mathrm{TM}}(\mathrm{SG})$ was introduced to assess individual responses to treatment. ${ }^{32}$ The $\mathrm{SG}$ provides descriptions of common dementia symptoms. In the version used 
here, 9-12 descriptors were provided for each of 27 symptoms, in a paper format. (There are descriptors for 60 symptoms in the on-line format: http://www.dementiaguide.com/symptomguide/) Caregivers completed the SG in an interview, facilitated by the CGI rater, where the caregivers chose from the list of symptoms those that were the most troubling to them. Next they ranked the symptoms, with the least troublesome ranked as 1 , and each other is ranked higher and their frequency of occurrence. The SG score is the average frequency of the symptoms in the individual with the weights represented by their ranks. As some troublesome symptoms can occur very frequently (e.g., many times a day in looking for a missing object, whereas others can occur less frequently, e.g., refusing to attend a regular weekly outing), the data were represented on a log scale to accommodate the large range, with the differences in the log scores between the visits represented graphically as the ratio of the last score to the baseline score. The SG method explicitly incorporates patient and caregiver input, which aids the understanding of clinical meaningfulness. ${ }^{33}$

Adverse events (AEs) that were observed, reported by the patients/caregivers, or elicited by direct questioning, were recorded as to severity, seriousness, cause and outcome.

\subsection{Analysis}

The efficacy analyses was based on the full analysis set sample, defined as all patients who received at least one dose of donepezil and who had baseline and at least one post-baseline assessment of efficacy. Missing data were imputed using the last observation carried forward (LOCF) endpoint, defined as the last assessment recorded post baseline. Note that both LOCF and observed case (OC) analyses were conducted. Neither method is without controversy, ${ }^{34,35}$ but given the emphasis here on detecting observed treatment effects, OC analyses are reported. The least squares (LS) mean and standard error were presented for variables analyzed with the Mixed Model. The covariates that were fitted as fixed effects in all the primary and secondary analyses were Center, Week, and Baseline score. The term subject was fitted as a random effects term. In addition to descriptive statistics, scores were compared between the baseline and 24 week visits, for patients who completed the study. Analysis of variance was used to compare continuous scores and chi-square for categorical variables. For the SG, which must combine frequencies across a large range, the non-parametric Mann-Whitney U test was used to analyse change. The CGI is a change score, so that the comparison measure is no change from baseline (or a score of " 4 ", where " 1 " = very much better, and " $7 "$ = very much worse). For all tests, a significant difference was set at $\mathrm{p}<0.05$.

To assess the clinical detectability of the various measures, standardized response means were calculated (where the mean before/after difference is divided by the standard deviation of the change). ${ }^{36}$ Interpretation is as with other standardized effect sizes. Here of most relevance is that the threshold for clinical detection is the Standard Response Mean (SRM) of 0.2; SRMs in the range of 0.2-0.4 are said to be small, ${ }^{37}$ which is common in trials of cholinesterase inhibitors in dementia. ${ }^{38}$ To assess their construct validity, the change scores were correlated (Pearson and Spearman correlation coefficients (when appropriate) were used) with each other and compared against the global clinical judgments.

\subsection{Ethics}

The ethics committees of each participating centre approved the protocol. A central ethics review was used for practices that were not affiliated with an academic centre. In addition, the protocol was approved by the Research Ethics Committee of the Capital District Health Authority, Halifax, NS and the Sunnybrook Health Science Centre for the sub-studies on the SymptomGuide $^{\mathrm{TM}}$ and imaging, respectively.

\section{Results}

\subsection{Baseline clinical characteristics}

Due to challenges with patient recruitment for this study, enrolment was stopped when 200 patients had been screened rather than extend the recruitment period even further to achieve the originally targeted 260 patients. Of the 200 screened patients, 148 received treatment and took at least one dose of donepezil.

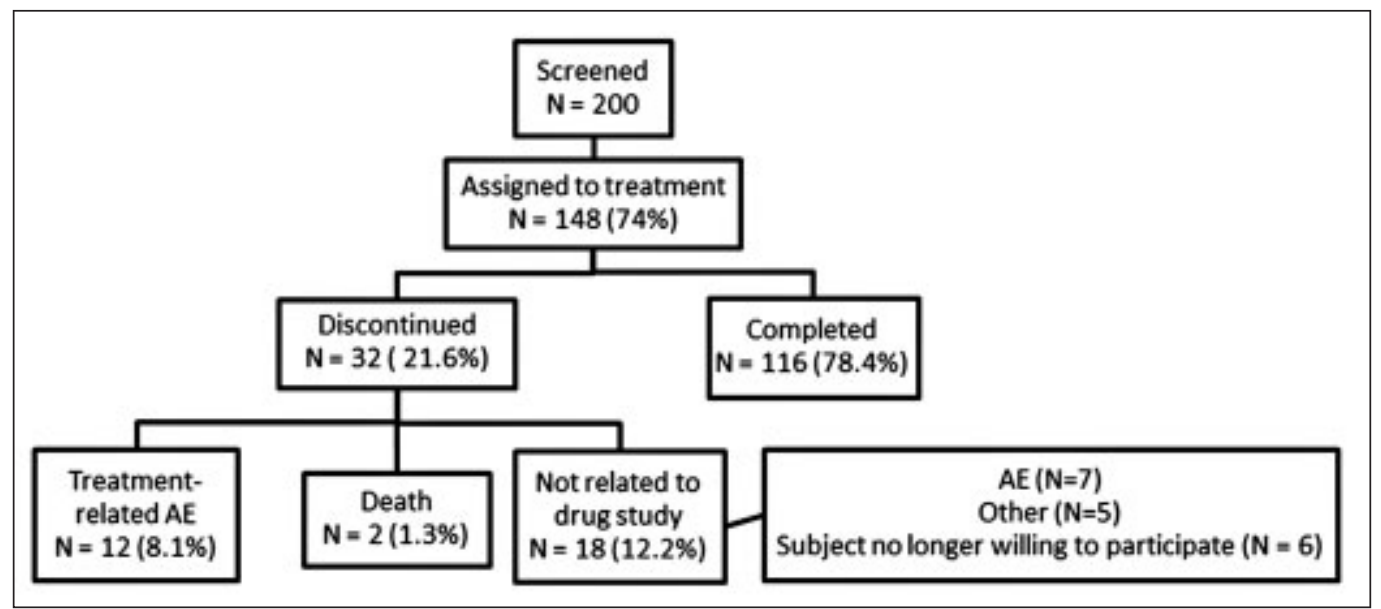

Figure 1: Subject disposition 
From these, 137 had at least one post-dose efficacy measure and 116 completed the study, for a drop-out rate of $21.6 \%$ (Figure 1). Patients who discontinued were slightly older, but otherwise were of similar demographic and clinical characteristics, including the proportion with $\mathrm{VaD}$ versus mixed $\mathrm{AD} / \mathrm{VaD}$. Overall, about half of the patients $(77 ; 52 \%)$ had a diagnosis of $\mathrm{VaD}$, with a mean duration of illness of 2.1 years. The 71 patients (48\%) with mixed $\mathrm{AD} / \mathrm{VaD}$ had a mean illness duration of 1.8 years. Ten patients had incomplete data at the final visit, although CGI data were available on all patients.

The patients were predominantly elderly (87\% were aged 65 years or older) with a slight female preponderance $(55 \%)$, although the male/female split was almost even in $\mathrm{VaD}$, compared with more women $(42 ; 62 \%)$ in mixed $\mathrm{AD} / \mathrm{VaD}$. Otherwise, the diagnostic subgroups had the same mean body mass index (BMI) (27.2). Clinically, the study population consisted mainly of people with mild dementia. The mean baseline sMMSE score was 23.4 , with $\mathrm{VaD}$ patients having a higher mean baseline sMMSE (24.8) than those with mixed $\mathrm{AD} / \mathrm{VaD}$ (22.0). By definition, the Hachinski Ischemia Scale (HIS) scores were higher in $\mathrm{VaD}$ than in mixed $\mathrm{AD} / \mathrm{VaD}$; overall, patients represented a range of ischemic injury, with most having a moderate ischemic burden (mean HIS $=8.0 \pm$ standard deviation (SD) 5.0). Otherwise, HAM_D-6 (mean 4.2 \pm 3.0 ), FAST (mean $4.3 \pm 0.5)$ and CGI-S $(3.4 \pm 0.4)$ were consistent with a group with mild-moderate dementia, as specified in the inclusion criteria. All patients reported at least one co-morbid illness. Vascular co-morbidities were the most common (total reported = 305), especially hypertension (present in 105 people), dyslipidemias (59) heart disease (43) and diabetes (30). Seventythree psychiatric co-morbidities were reported, chiefly anxiety (17) and depression (28). Patients were using one to two drugs on average at baseline; the most common drug classes were
Table 1: Caregiver characteristics

\begin{tabular}{lcc}
\hline Caregiver Characteristics & VaD (N=77) & Mixed AD/VaD (N=71) \\
\hline Mean Age (SD) & $66.1(12.8)$ & $64.6(3.9)$ \\
Gender (\% female) & $68 \%$ & $71 \%$ \\
Mean Education years (SD) & $12.7(3.5)$ & $12.9(3.9)$ \\
$\%$ Living with subject & $83 \%$ & $53 \%$ \\
Relationship with subject & & \\
$\quad$ Spouse & $72 \%$ & $53 \%$ \\
Son/Daughter & $23 \%$ & $39 \%$ \\
Friend/Professional Caregiver & $5 \%$ & $8 \%$ \\
Employment status & & \\
Retired & $64 \%$ & $58 \%$ \\
Full Time & $16 \%$ & $22 \%$ \\
Homemaker & $10 \%$ & $2 \%$ \\
Part Time & $4 \%$ & $15 \%$ \\
Unemployed/Other & $3 \%$ & $2 \%$ \\
\hline
\end{tabular}

$\mathrm{AD}$ - Alzheimer's disease; SD - standard deviation; VaD - vascular dementia

antithrombotic agents (39), analgesics (38), and antihypertensives (37).

The majority of the caregivers were women (Table 1). Most patients were cared for by a spouse and most were retired.

\subsection{Efficacy measures}

Regarding the primary efficacy measure, although the sMMSE scores increased slightly at Week 24 (LS mean change $=0.72$, Table 2 ), this difference was not statistically significant $(\mathrm{p}=0.06$, Table 2$)$ and the effect size was small $(\mathrm{SRM}=0.15$,

Table 2: Scores of standardized outcome measures (mean, standard deviation, range and sample size) from baseline to week 24

\begin{tabular}{|c|c|c|c|c|c|}
\hline Measure & Primary Diagnosis & Baseline & Week 12 & Week 24 & P value \\
\hline \multirow[t]{2}{*}{ SMMSE } & All Subjects & $23.4(4.5 ; 10-30)$ & $23.98(4.6 ; 10-30)$ & $24.12(4.7 ; 11-30)$ & 0.06 \\
\hline & & $\mathrm{N}=137$ & $\mathrm{~N}=124$ & $\mathrm{~N}=114$ & \\
\hline \multirow[t]{2}{*}{ DAD } & All Subjects & $80.1(18.6 ; 25-100)$ & $81.4(20.3 ; 10-106)$ & $78.9(23.6 ; 9.5-100)$ & 0.90 \\
\hline & & $\mathrm{N}=137$ & $\mathrm{~N}=124$ & $\mathrm{~N}=114$ & \\
\hline \multirow[t]{2}{*}{ NPI-Q } & All Subjects & $6.8(5.4 ; 0-28)$ & $5.4(4.7 ; 0-23)$ & $5.4(5.1 ; 0-30)$ & 0.01 \\
\hline & & $\mathrm{N}=137$ & $\mathrm{~N}=124$ & $\mathrm{~N}=114$ & \\
\hline \multirow[t]{2}{*}{ NPI-Q-D } & All Subjects & $7.4(6.8 ; 0-30)$ & $6.1(6.6 ; 0-35)$ & $5.8(6.9 ; 0-41)$ & 0.11 \\
\hline & & $\mathrm{N}=137$ & $\mathrm{~N}=124$ & $\mathrm{~N}=114$ & \\
\hline \multirow[t]{2}{*}{ CLOX -1 } & All Subjects & $8.7(3.9 ; 0-15)$ & $8.7(4.1 ; 0-15)$ & $9.6(3.9 ; 0-15)$ & 0.006 \\
\hline & & $\mathrm{N}=137$ & $\mathrm{~N}=123$ & $\mathrm{~N}=111$ & \\
\hline \multirow[t]{6}{*}{ CLOX -2 } & All Subjects & $11.2(3.2 ; 0-12)$ & $11.5(3.4 ; 0-15)$ & $12.1(2.9 ; 0-15)$ & 0.03 \\
\hline & & $\mathrm{N}=131$ & $\mathrm{~N}=117$ & $\mathrm{~N}=106$ & \\
\hline & $\mathrm{VaD}$ & $11.6(3.1 ; 0-15)$ & $11.8(3.3 ; 0-15)$ & $12.6(2.4 ; 4-15)$ & 0.12 \\
\hline & & $\mathrm{N}=67$ & $\mathrm{~N}=60$ & $\mathrm{~N}=56$ & \\
\hline & Mixed AD/VaD & $11.6(3.4 ; 0-15)$ & $11.2(3.6 ; 0-15)$ & $12.6(2.4 ; 4-15)$ & 0.03 \\
\hline & & $\mathrm{N}=50$ & $\mathrm{~N}=57$ & $\mathrm{~N}=56$ & \\
\hline \multirow[t]{6}{*}{ Phonetic Fluency } & All Subjects & $7.4(3.7 ; 0-17)$ & $8.1(4.1 ; 0-18)$ & $8.3(4.2 ; 0-21)$ & 0.01 \\
\hline & & $\mathrm{N}=136$ & $\mathrm{~N}=123$ & $\mathrm{~N}=113$ & \\
\hline & $\mathrm{VaD}$ & $8.2(3.5 ; 1-17)$ & $9.1(3.9 ; 1-17)$ & $8.9(4.1 ; 2-21) \mathrm{N}=68$ & 0.39 \\
\hline & & $\mathrm{N}=68$ & $\mathrm{~N}=62$ & & \\
\hline & Mixed AD/VaD & $6.6(3.7 ; 0-16)$ & $7.2(4.2 ; 0-18)$ & $7.6(4.1 ; 0-17) \mathrm{N}=55$ & 0.01 \\
\hline & & $\mathrm{N}=68$ & $\mathrm{~N}=61$ & & \\
\hline
\end{tabular}

CLOX-1 - clock drawing (hands free); CLOX-2 - clock drawing (copied); DAD - Disability Assessment for Dementa; NPI-Q - brief Neuropsychiatric Inventory; NPI-Q-D - brief Neuropsychiatric Inventory Distress; sMMSe - standardised Mini-Mental State Examination; VaD - vascular dementia 
Table 3: Comparative responsiveness of outcome measures (mean, standard deviation, range and sample size)

\begin{tabular}{lccc}
\hline Measure & Baseline & Week 24 & SRM \\
SMMSE & $23.4(4.5 ; 10-30) \mathrm{N}=137$ & $24.12(4.7 ; 11-30) \mathrm{N}=114$ & 0.15 \\
DAD & $80.1(18.6 ; 25-100) \mathrm{N}=137$ & $78.9(23.6 ; 9.5-100) \mathrm{N}=114$ & -0.05 \\
NPI-Q & $6.8(5.4 ; 0-28) \mathrm{N}=137$ & $5.4(5.1 ; 0-30) \mathrm{N}=114$ & -0.24 \\
NPI-Q-D & $\mathbf{7 . 4 ( 6 . 8 ; 0 - 3 0 ) ~ N = 1 3 7}$ & $\mathbf{5 . 8}(\mathbf{6 . 9} ; \mathbf{0 - 4 1}) \mathbf{N = 1 1 4}$ & $\mathbf{- 0 . 2 3}$ \\
CLOX -1 & $8.7(3.9 ; 0-15) \mathrm{N}=137$ & $9.6(3.9 ; 0-15) \mathrm{N}=111$ & 0.22 \\
CLOX -2 & $11.2(3.2 ; 0-12) \mathrm{N}=131$ & $12.1(2.9 ; 0-15) \mathrm{N}=106$ & 0.26 \\
Phonetic Fluency & $7.4(3.7 ; 0-17) \mathrm{N}=136$ & $8.3(4.2 ; 0-21) \mathrm{N}=113$ & 0.21 \\
Symptom Guide & $-2.7(1.5 ;-5.2-2) \mathrm{N}=128$ & $-2.9(1.7 ;-6.3-2) \mathrm{N}=106$ & -0.28 \\
\hline
\end{tabular}

CLOX-1 - clock drawing (hands free); CLOX-2 - clock drawing (copied); DAD - Disability Assessment for Dementa; NPI-Q - brief Neuropsychiatric Inventory; NPI-Q-D - brief Neuropsychiatric Inventory Distress; sMMSe - standardised Mini-Mental State Examination; SRM - Standardized Response Mean

Table 3). Apart from CLOX-2 and Phonetic Fluency (Table 2), there was no difference in results between the mixed AD/VaD patients and the $\mathrm{VaD}$ patients (data not shown). The percentage of patients with no response (a change from baseline of zero or less) on the sMMSE decreased from $47.5 \%$ at Week 12 to $40.1 \%$ at Week 24. After six months of treatment with donepezil, the DAD showed no statistically significant difference (Table 2) with a very small effect size $(\mathrm{SRM}=-0.05$, Table 3$)$. Likewise, the NPI-Q-D scores were not significantly better.

Other tests were more responsive. The CLOX-1 and CLOX2 tests both showed statistically significantly better performance (Table 2), although with small effect sizes (Table 3). The NPI-Q similarly recorded significantly fewer disturbances (Table 2) with small effect sizes (Table 3). The Phonetic Fluency test showed statistically significant increases after six months (Table 2) with small, yet detectable effect sizes (SRM $=0.21$, Table 3$)$.

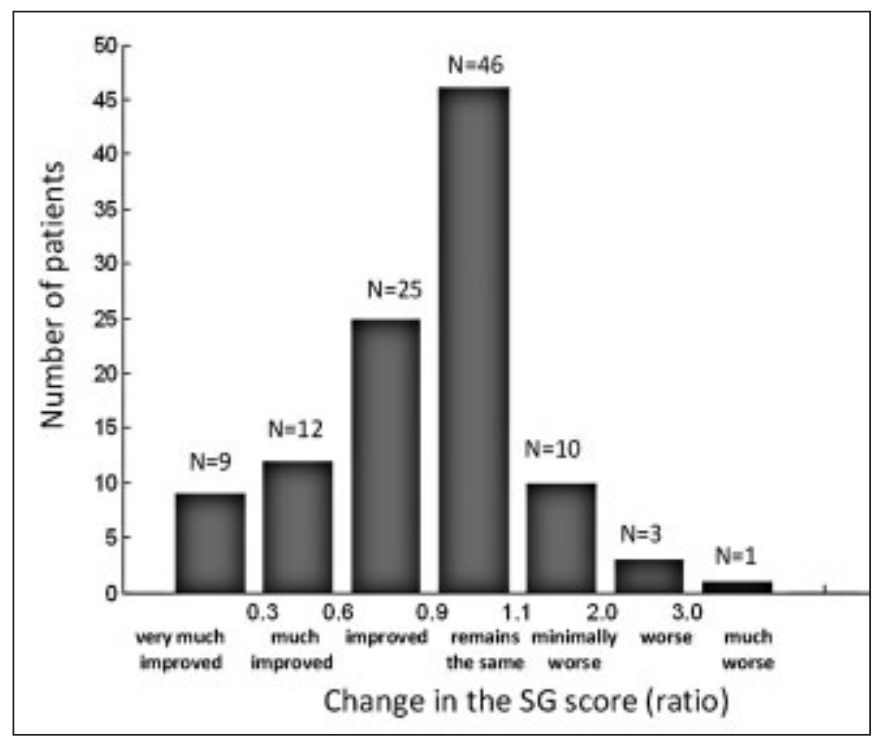

Figure 2: Change in SymptomGuide (SG) score from baseline visit to last visit
The SG showed statistically significant improvement with donepezil treatment (Figure 2). For example, it shows some improvement (with the ratio up to two times) in 46 patients, with worsening occurring in 14 patients. The effect size of the differences were small ( $\mathrm{SRM}=-0.28$, Table 3$)$. The mean change in the CGI was 3.32 (95\% confidence interval $=3.10-3.54$ ). Similarly, the most common CGI-I response was improvement, mostly "minimal" improvement (Figure 3). The Spearman correlation between the changes in the SG (expressed by the ratio SG at the last week to the baseline visit) and CGI was 0.32 $(p=0.001)$. Although overall the executive function and judgment based measures each point in this direction (Table 3) the individual correlation coefficients between measures was small (range -0.22 to 0.30 ).

\subsection{Safety and adverse effects}

Three hundred and sixty-three adverse events were recorded in the 148 patients, of which 130 were felt to be related to treatment (Table 4a). Adverse events (AE) were defined as any

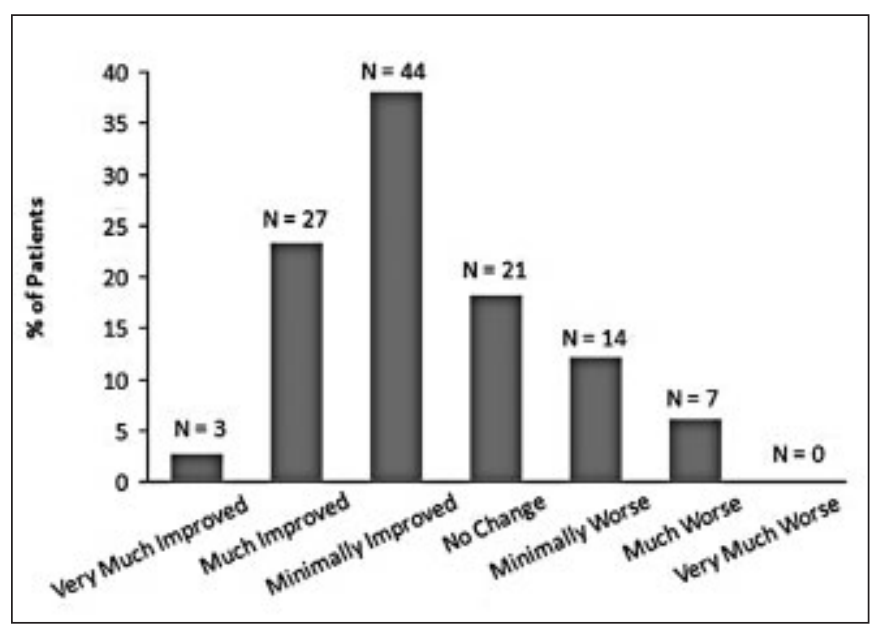

Figure 3: Proportion of patients by Clinical Global Impression at Week 24 
Table 4a: Overview of adverse events (AE)

\begin{tabular}{lcc}
\hline & Number of Patients & Donepezil N $=\mathbf{1 4 8}$ \\
\cline { 2 - 2 } & All Causalities & Treatment-Related \\
Patients evaluable for AEs & N $(\%)$ & 148 \\
Patients with AEs & $110(74.3)$ & $62(41.9)$ \\
Patients with serious AEs & $24(16.2)$ & $2(1.4)$ \\
Patients with severe AEs & $22(14.9)$ & $4(2.7)$ \\
Patients discontinued due to AEs & $20(13.5)$ & $12(8.1)$ \\
Patients with dose reduced or temporary discontinuations due to AEs & $24(16.2)$ \\
\hline
\end{tabular}

untoward medical occurrence in a patient administered an investigational product or medical device. Serious adverse events (SAE) were defined as any untoward medical occurrence at any dose that results in death, is life threatening, requires inpatient hospitalization or prolongation of existing hospitalization, results in significant or persistent disability/ incapacity or results in congenial abnormality or birth defect. All adverse events were rated for severity (mild, moderate, severe) by the investigating physician. During the course of this study, two patients $(1.4 \%)$ died. One patient had two strokes, one in the active treatment period and one during the post treatment period. The second patient died following a worsening of general health status. None of these three events or two deaths was considered by the investigator as related to study drug. Fewer than half of the patients $(41.9 \%)$ reported treatment-related AEs (Table $4 a)$. Few patients reported treatment-related serious and severe AEs (1.4\% and $2.7 \%$, respectively). Twenty patients (13.5\%) permanently discontinued the study due to an AE (all causalities), whereas 24 patients $(16.2 \%)$ had to either reduce their dosage or temporarily discontinue due to AEs.

The most common treatment-related AEs (Table 4b) were gastrointestinal disorders, reported in 36 patients (24\%), chiefly nausea $(14.9 \%)$ and diarrhea $(8.1 \%)$. Nervous system disorders were reported by 16 patients $(10.8 \%)$ and psychiatric disorders by $9.5 \%$ of patients.

Table 4b: Frequency and severity of treatment-related adverse events

\begin{tabular}{lcccc}
\hline \multirow{2}{*}{ System Organ Class Preferred Term } & \multicolumn{3}{c}{ Donepezil (n=148) } \\
\cline { 2 - 5 } & $\mathbf{N}(\boldsymbol{\%})$ & $\underline{\text { Mild }}$ & $\underline{\text { Moderate }}$ & $\underline{\text { Severe }}$ \\
Gastrointestinal disorders & $36(24.3)$ & 15 & 21 & 0 \\
Diarrhea & $12(8.1)$ & 4 & 8 & 0 \\
Nausea & $22(14.9)$ & 13 & 9 & 0 \\
Vomiting & $7(4.7)$ & 5 & 2 & 0 \\
General disorders & $7(4.7)$ & 3 & 4 & 0 \\
Metabolism and nutrition disorders & $7(4.7)$ & 3 & 3 & 1 \\
Nervous system disorders & $16(10.8)$ & 8 & 8 & 0 \\
Dizziness & $8(5.4)$ & 5 & 3 & 0 \\
Psychiatric disorders & $14(9.5)$ & 6 & 6 & 2 \\
Insomnia & $8(5.4)$ & 5 & 2 & 1 \\
\hline
\end{tabular}

\section{Discussion}

In this six-month study, several measures that might feasibly be employed in primary care (such as the sMMSE, CLOX tests and $\mathrm{SG}$ ) of patients with $\mathrm{VaD}$ and mixed $\mathrm{AD} / \mathrm{VaD}$ showed the varying interpretations of change following treatment with donepezil. On the one hand, there was no statistically significant change in the sMMSE or activities of daily living; change in behaviour seemed to vary depending on the measure. On the other hand, there appears to have been some overall clinical benefit, as suggested by both individualized tests (CGI and the SG) and by those standardized tests that measured executive function (CLOX-1, CLOX-2, and Phonetic Fluency). This latter observation is potentially clinically important, given the role of executive dysfunction across the VCI spectrum ${ }^{1,2,18,19,39}$.

The data must be interpreted with caution as due to recruitment difficulties the sample size was less than aimed for. With 116 completers, we estimate a $34 \%$ chance of type II error (missing a significant difference) in the sMMSE. In addition, generalizability may not be assured given that the VCI construct was operationalized here for two subcomponents ( $\mathrm{VaD}$ and mixed $\mathrm{AD} / \mathrm{VaD}$ ) which may not be widely used in all primary care settings. We underscore that the open-label design does not allow us to attribute change (or lack of it) to treatment with donepezil. An open-label trial, however, is appropriate for the evaluation of measurement issues, where the design can be viewed as a setting in which patients and treatment have been held constant, but measures varied. In consequence, we have been careful to keep inferences to that - i.e. measurement, not efficacy.

Of note, most patients did not deteriorate over the six months, so the magnitude of change is mostly in the "clinically detectable, but small" range 37,38 . Other factors associated with participation in a clinical trial - e.g., selection, vascular risk factor control, the placebo response, learning effects, chance might account for the stability seen in most patients. Whether the response is maintained, worsened or enhanced over a longer period is not clear. With regard to safety, all enrolled subjects were analyzed for AEs, vital signs data, and ECG data. Laboratory parameters were analyzed for subjects who received at least one dose of donepezil and who had baseline and at least one post-baseline laboratory assessment $(n=125$ [84.5\%]). Based on the advanced age and underlying medical condition of the 
subjects enrolled in this study, the reported AEs and abnormal laboratory values were not unexpected.

Several lines of evidence, in addition to SRM estimates, point to clinical change on average having been detectable, but small. Here, the SG showed an effect size of -0.28 (between the baseline and week 24 visits). The SG's summative intensity score (combining symptom frequency and the rank of its importance) for all symptoms corresponds to approximately 1.7 times a day for their occurrence at baseline, and about 1.2 times a day at week 24 . This amounts to about an average $33 \%$ decrease in symptom frequency per individual, which on its face would seem to be both detectable and, on average, clinically meaningful, even if small. This analysis of a symptom-based approach to measuring treatment effects is in accord with a review which concluded that, despite their being central to the interpretation of trial results and to decisions regarding whether to employ trial findings in clinical practice, patient- and caregiver-centered measures of clinical significance have not been adequately studied ${ }^{33}$. Here, inspection of the distribution of the scores suggests that while most patients showed only a small change, the average effect reflects that a few patients showed a very large change - typically in the significant diminution of common and troubling symptoms. Likewise, a few patients showed important worsening, although on average more patients showed large improvement rather than large decline. While it might be that such stability at six months is better than expected, the clinical trial experience in $\mathrm{VaD}$ often shows placebo patients with little decline at six months ${ }^{11,16,22}$.

These data lend support to the contention that some part of the controversy over the effects of cholinesterase inhibitor treatment reflects how outcomes are measured. Many clinicians and caregiver advocacy groups have felt persuaded that they can see meaningful benefit from treatment. But if what they see has little chance to be captured by standard clinical trials instruments, there is no way for such evidence to be adjudicated ${ }^{40}$. Given how many new drugs for dementia are being tested, it remains useful to measure benefits which are likely to be both important and clinically meaningful, and which might capture unexpected benefits arising from novel approaches to treatment. Despite the intensity of effort and resources being put into them, as argued elsewhere $^{32}$ it is doubtful that the emphasis on biomarkers as outcome measures will meet the need to demonstrate clinically meaningful change without, at the very least, being judged against clinically meaningful outcomes. For this reason, there remains a role for studies which address how to detect changes that might be associated with drug treatment, especially where clinical experience suggests that current measures are missing useful clinical information ${ }^{41-44}$. In Canada, this may have particular relevance to provincial formularies, many of which will only reimburse the cost of dementia treatment to people whose Mini-Mental State Examination test scores improve. Further work on which symptoms are most responsive, which are most resistant to change, or how any such patterns might be localized, are intriguing questions that are motivating further inquiries.

\section{ACKNOWLEDGMENTS}

Kenneth Rockwood is supported by the Dalhousie Medical Research Foundation as the Kathryn Allen Weldon Professor of
Alzheimer Research. Sandra Black is supported by the Neuroscience Research Program and the Department of Medicine, Sunnybrook Health Sciences Centre, University of Toronto as the Brill Professor of Neurology at the University of Toronto.

The authors would like to acknowledge Isabelle Lussier, $\mathrm{PhD}$ and David A Gold, PhD for the initial development of this study, Marc-André Bédard, $\mathrm{PhD}$ for his assistance during the conduct of the study, and Kalliopi Dimos, BSc for the management of the conduct of this study, as well as An Zeng $\mathrm{PhD}$ and the late Yikang $\mathrm{Xu}, \mathrm{PhD}$ for statistical support and review of earlier drafts of this manuscript.

\section{Role OF THE Authors}

KR: Was a paid consultant to Pfizer Canada for this study and, as such, developed the research protocol with Pfizer Canada, trained participating physicians, wrote the first draft of the manuscript and revised subsequent drafts. KR had access to all of the data. The SG data reside fully with KR and he was responsible for their analyses.

SEB: Was a paid consultant to Pfizer Canada for this study and, as such, developed the research protocol with Pfizer Canada, wrote imaging protocol, trained participating physicians and reviewed and contributed to the manuscript. SEB had access to all of the data. The imaging data resides fully with SEB and she was responsible for their analysis.

AM: Supervised the analyses and designed analyses for the SG.

MR: Conducted analyses on SG data, held in-house at DGI Clinical Inc.

ID: Supervised the development of the statistical analysis plan, analyses of the data and interpretation of the data. ID reviewed and contributed to the manuscript.

\section{CONFLICT OF INTEREST}

Kenneth Rockwood was paid as consultant to this project. In addition, KR is President, Chief Scientific Officer and a shareholder in DGI Clinical Inc. (formerly DementiaGuide Inc.), which owns the SymptomGuide ${ }^{\mathrm{TM}}$ that was employed as an outcome measure in this trial. In the last five years, KR has also sat on advisory boards for Pfizer Canada, Glaxo SmithKline and Elan/Wyeth, and given a talk sponsored by Bristol Myers Squib.

Sandra E. Black was paid as consultant to this project. In addition, $\mathrm{SB}$ receives honoraria from $\mathrm{CME}$ and ad hoc consulting from Pfizer, Janssen-Ortho, Novartis Pharmaceuticals, Lundbeck and Myriad Pharmaceuticals and operating funds from Pfizer Inc., Janssen Ortho, Novartis Pharmaceuticals, Myriad Pharmaceuticals, Sanofi-Aventis, Astra-Zeneca, Boehringer Ingelheim and Nova Nordisk.

Isabelle Defoy is an employee of Pfizer Canada Inc. This study was sponsored and managed by Pfizer Canada Inc.

\section{REFERENCES}

1. Moorhouse P, Rockwood K. Vascular cognitive impairment: current concepts and clinical developments. Lancet Neurol. 2008;7: 246-55.

2. Gorelick PB, Scuteri A, Black SE, et al. Vascular contributions to cognitive impairment and dementia: a statement for healthcare professionals from the American Heart Association/American Stroke Association. Stroke. 2011;42:2672-713. 
3. Román GC, Tatemichi TK, Erkinjuntti T, et al. Vascular dementia: diagnostic criteria for research studies. Report of the NINDSAIREN International Workshop. Neurology. 1993;43:250-60.

4. Wang BW, Lu E, Mackenzie IR, et al. Multiple pathologies are common in Alzheimer patients in clinical trials. Can J Neurol Sci. 2012;39(5):592-9.

5. Snowdon DA, Greiner LH, Mortimer JA, Riley KP, Greiner PA, Markesbery WR. Brain infarction and the clinical expression of Alzheimer disease. The Nun Study. JAMA 1997;277:813-7.

6. Neuropathology Group. Medical Research Council Cognitive Function and Aging Study. Pathological correlates of late-onset dementia in a multicentre, community-based population in England and Wales. Neuropathology Group of the Medical Research Council Cognitive Function and Ageing Study (MRC CFAS). Lancet. 2001;357:169-75.

7. Launer LJ, Petrovitch H, Webster RG, Markesbery W, White LR. AD brain pathology: vascular origins? Results from the HAAS autopsy study. Neurobiol Aging. 2008;29:1587-90.

8. Shi GX, Liu CZ, Wang LP, Guan LP, Li SQ. Biomarkers of oxidative stress in vascular dementia patients. Can J Neurol Sci. 2012;39(1):65-8

9. Perry G, Phelix CF, Nunomura A, et al. Untangling the vascular web from Alzheimer disease and oxidative stress. Can J Neurol Sci. 2012;39(1):4

10. Birks J. The evidence for the efficacy of cholinesterase inhibitors in the treatment of Alzheimer's disease is convincing. Int Psychogeriatr. 2008;20:1-7.

11. Erkinjuntti T, Roman G, Gauthier S, Feldman H, Rockwood K. Emerging therapies for vascular dementia and vascular cognitive impairment. Stroke. 2004;35:1010-17.

12. Wong CL, Bansback N, Lee PE, Anis AH. Cost-effectiveness: cholinesterase inhibitors and memantine in vascular dementia. Can J Neurol Sci. 2009;36(6):735-9.

13. Wilkinson D, Doody R, Helme R, et al. Donepezil in vascular dementia: a randomized, placebo-controlled study. Neurology. 2003;61:479-86

14. Black S, Román GC, Geldmacher DS, et al. Donepezil 307 Vascular Dementia Study Group. Efficacy and tolerability of donepezil in vascular dementia: positive results of a 24 -week, multicenter, international, randomized, placebo-controlled clinical trial. Stroke. 2003;34:2323-30.

15. Mohs RC, Knopman D, Petersen RC, et al. Development of cognitive instruments for use in clinical trials of antidementia drugs: additions to the Alzheimer's Disease Assessment Scale that broaden its scope. The Alzheimer's Disease Cooperative Study. Alzheimer Dis Assoc Disord. 1997;11(suppl 2):S13-21.

16. Román GC, Salloway S, Black SE, et al. Randomized, placebocontrolled, clinical trial of donepezil in vascular dementia: differential effects by hippocampal size. Stroke. 2010;41: 1213-21.

17. Gauthier S, Patterson C, Chertkow H, et al. Recommendations of the 4th Canadian Consensus Conference on the Diagnosis and Treatment of Dementia (CCCDTD4). Can Geriatr J. 2012 Dec; 15(4):120-6.

18. Levine DA, Langa KM. Vascular cognitive impairment: disease mechanisms and therapeutic implications. Neurotherapeutics. 2011;8:361-73

19. Gauthier S, Ferris S. Outcome measures for probable vascular dementia and Alzheimer's disease with cerebrovascular disease. Int J Clin Pract Suppl. 2001;(120):29-39.

20. Winblad B, Engedal K, Soininen H, et al. A 1-year, randomized, placebo-controlled study of donepezil in patients with mild to moderate AD. Neurology. 2001;57:489-95.

21. Román GC, Wilkinson DG, Doody RS, Black SE, Salloway SP, Schindler RJ. Donepezil in vascular dementia: combined analysis of two large-scale clinical trials. Dement Geriatr Cogn Disord. 2005;20:338-44.

22. American Psychiatric Association. Diagnostic and Statistical Manual. 4th ed., text rev. Washington: American Psychiatric Association; 2005.

23. Hachinski VC, Iliff LD, Zilhka E, et al. Cerebral blood flow in dementia. Arch Neurol. 1975;32:632-7.
24. Reisberg B. Functional assessment staging (FAST). Psychopharmacol Bull. 1988;24:653-9.

25. Rockwood K, Black SE, Song X, et al. Clinical and radiographic subtypes of vascular cognitive impairment in a clinic-based cohort study. J Neurol Sci. 2006;240(1-2):7-14

26. Hamilton M. A rating scale for depression. J Neurol Neurosurg Psychiatry. 1960;23:56-62.

27. Molloy DW, Standish TI. A guide to the standardized Mini-Mental State Examination. Int Psychogeriatr. 1997;9(Suppl 1):87-94; discussion 143-50

28. Gélinas I, Gauthier L, McIntyre M, Gauthier S. Development of a functional measure for persons with Alzheimer's disease: the disability assessment for dementia. Am J Occup Ther. 1999 SepOct;53(5):471-81.

29. Royall DR, Cordes JA, Polk M. CLOX: an executive clock drawing task. J Neurol Neurosurg Psychiatry. 1998;64:588-94.

30. Gladsjo JA, Schuman CC, Evans JD, Peavy GM, Miller SW, Heaton RK. Norms for letter and category fluency: demographic corrections for age, education, and ethnicity. Assessment. 1999; $147-78$

31. Kaufer DI, Cummings JL, Ketchel P, et al. Validation of the NPI-Q, a brief clinical form of the Neuropsychiatric Inventory. J Neuropsychiatry Clin Neurosci. 2000;12:233-9.

32. Rockwood K. An individualized approach to tracking and treating Alzheimer's disease. Clin Pharmacol Ther. 2010;88:446-9.

33. Molnar FJ, Man-Son-Hing M, Fergusson D. Systematic review of measures of clinical significance employed in randomized controlled trials of drugs for dementia. J Am Geriatr Soc. 2009; 57:536-46.

34. Molnar FJ, Man-Son-Hing M, Hutton B, Fergusson DA. Have lastobservation-carried-forward analyses caused us to favour more toxic dementia therapies over less toxic alternatives? A systematic review. Open Med. 2009;3(2):e31-50.

35. Little RJ, D'Agostino R, Cohen ML, et al. The prevention and treatment of missing data in clinical trials. N Engl J Med. 2012; 367(14):1355-60.

36. Liang MH, Fossel AH, Larson MG. Comparisons of five health status instruments for orthopedic evaluation. Med Care. 1990; 28:632-42.

37. Cohen J. Statistical Power Analysis for the Behavioral Sciences. 2nd ed. Englewood Cliffs: Lawrence Erlbaum Associates; 1988.

38. Rockwood K. Size of the treatment effect on cognition of cholinesterase inhibition in Alzheimer's disease. J Neurol Neurosurg Psychiatry. 2004;75(5):677-85.

39. Moorhouse P, Song X, Rockwood K, et al., Executive dysfunction in vascular cognitive impairment in the consortium to investigate vascular impairment of cognition study. J Neurol Sci. 2010;288:142-6.

40. Rockwood K. Should we listen to people affected by dementia? CNS Neurosci Ther. 2011;17 (1):1-3.

41. Ballard C, Gauthier S, Corbett A, Brayne C, Aarsland D, Jones E. Alzheimer's disease. Lancet. 2011;377(9770):1019-31.

42. Rockwood K, Black SE, Robillard A, Lussier I. Potential treatment effects of donepezil not detected in Alzheimer's disease clinical trials: a physician survey. Int J Geriatr Psychiatry. 2004;19(10): 954-60.

43. Behl P, Lanctôt KL, Streiner DL, Black SE. The effect of cholinesterase inhibitors on decline in multiple functional domains in Alzheimer's disease: a two-year observational study in the Sunnybrook dementia cohort. Int Psychogeriatr. 2008;20: 1141-59.

44. Gillette-Guyonnet S, Andrieu S, Nourhashemi F, et al. REAL.FR study group. Long-term progression of Alzheimer's disease in patients under antidementia drugs. Alzheimers Dement. 2011;7 (6):579-92. 